University of New Hampshire

University of New Hampshire Scholars' Repository

Space Science Center

Institute for the Study of Earth, Oceans, and

Space (EOS)

9-26-2013

\title{
Scintillator gamma-ray detectors with silicon photomultiplier readouts for high-energy astronomy
}

\author{
Peter F. Bloser \\ University of New Hampshire, Peter.Bloser@unh.edu \\ Jason S. Legere \\ University of New Hampshire, jslegere@unh.edu \\ Chris Bancroft \\ University of New Hampshire \\ Mark L. McConnell \\ University of New Hampshire - Main Campus, mark.mcconnell@unh.edu \\ James M. Ryan \\ University of New Hampshire, James.Ryan@unh.edu
}

See next page for additional authors

Follow this and additional works at: https://scholars.unh.edu/ssc

Part of the Astrophysics and Astronomy Commons

\section{Recommended Citation}

Citation Peter F. Bloser ; Jason Legere ; Christopher Bancroft ; Mark L. McConnell ; James M. Ryan and Nathan Schwadron " Scintillator gamma-ray detectors with silicon photomultiplier readouts for highenergy astronomy ", Proc. SPIE 8859, UV, X-Ray, and Gamma-Ray Space Instrumentation for Astronomy XVIII, 88590A (September 26, 2013); doi:10.1117/12.2024411; http://dx.doi.org/10.1117/12.2024411

This Conference Proceeding is brought to you for free and open access by the Institute for the Study of Earth, Oceans, and Space (EOS) at University of New Hampshire Scholars' Repository. It has been accepted for inclusion in Space Science Center by an authorized administrator of University of New Hampshire Scholars' Repository. For more information, please contact Scholarly.Communication@unh.edu. 


\section{Authors}

Peter F. Bloser, Jason S. Legere, Chris Bancroft, Mark L. McConnell, James M. Ryan, and Nathan Schwadron 


\title{
Scintillator Gamma-Ray Detectors with Silicon Photomultiplier Readouts for High-Energy Astronomy
}

\author{
Peter F. Bloser*, Jason Legere, Christopher Bancroft, Mark L. McConnell, \\ James M. Ryan, and Nathan Schwadron \\ Space Science Center, University of New Hampshire, Durham, NH 03824
}

\begin{abstract}
Space-based gamma-ray detectors for high-energy astronomy face strict constraints of mass, volume, and power, and must endure harsh operating environments. Scintillator materials have a long history of successful operation under these conditions, and new materials offer greatly improved performance in terms of efficiency, time response, and energy resolution. The use of scintillators in space remains constrained, however, by the mass, volume, and fragility of the associated light readout device, typically a vacuum photomultiplier tube (PMT). Recently developed silicon photomultipliers (SiPMs) offer gains and efficiencies similar to those of PMTs, but with greatly reduced mass and volume, high ruggedness, and no high-voltage requirements. We have therefore been investigating the use of SiPM readouts for scintillator gamma-ray detectors, with an emphasis on their suitability for space- and balloonbased instruments for high-energy astronomy. We present our most recent results, including spectroscopy measurements for lanthanum bromide scintillators with SiPM readouts, and pulse-shape discrimination using organic scintillators with SiPM readouts. We also describe potential applications of SiPM readouts to specific highenergy astronomy instrument concepts.
\end{abstract}

Keywords: Silicon Photomultipliers; Scintillators; $\mathrm{LaBr}_{3}$; Gamma-ray Spectroscopy; High-energy astronomy

\section{INTRODUCTION}

Recent advances in scintillator technology, such as the availability of $\operatorname{LaBr}_{3}{ }^{1}$, have opened up a wide range of exciting new possibilities for high-energy astronomy and space science ${ }^{2-5} . \mathrm{LaBr}_{3}$ offers an energy resolution for gamma-ray measurement that is competitive with that of semiconductor detectors while retaining the many strengths of scintillators: simple and reliable implementation, high stopping power, large volume, room temperature operation, and very fast timing. In addition, new growth techniques have greatly increased the utility of organic scintillator crystals, such as stilbene, that feature high light output and good differentiation between electron and proton signals via pulse-shape discrimination (PSD) ${ }^{6}$. Advanced scintillators such as these could enable sensitive new instruments, such as a fast scintillator Compton telescope with greatly improved sensitivity over previous instruments ${ }^{7}$. The advantages of a scintillator are limited, however, by the volume, mass, and power of the associated light readout device, traditionally a vacuum photo-multiplier tube (PMT). Although PMTs have a long history of successful performance in space missions, they are inherently large, heavy, and fragile.

In recent years, Silicon Photomultiplier (SiPM) devices have been developed that offer a robust, low-mass, lowvolume alternative to PMTs. Originally developed for high-energy physics applications ${ }^{8}$, SiPMs are currently undergoing rapid development for use in nuclear medicine detectors; the combination of SiPMs with advanced scintillators such as $\mathrm{LaBr}_{3}$ is one area of intense investigation'. SiPMs consist of two-dimensional arrays of small $(\sim 50 \mu \mathrm{m})$ avalanche photodiode elements, or "cells," that are operated in limited Geiger mode and read out in parallel. Each cell includes an integrated resistor to quench the avalanche after several tens of nanoseconds. The summed output signal is proportional to the total number of cells that are triggered by the absorption of an optical photon. When coupled to a scintillator, a SiPM will therefore measure the brightness of the optical pulse generated by a gamma-ray interaction, as long as the number of cells is significantly greater than the number of incident scintillation photons. This combination of proportional response with Geiger-mode avalanche operation results in a solid-state light detector with high gain $\left(\sim 10^{6}\right)$ and fast time response (rise time of $\sim$ several ns, although this depends on the size and readout circuit), nearly equivalent to a PMT. The overall photon detection efficiency (PDE) is typically $20 \%-30 \%$, equivalent to a PMT.

Replacing PMTs with SiPM readouts for scintillators would offer many valuable advantages for space flight applications, including Compton telescopes. Similar photon detection efficiency, gain, and timing response could be achieved with a device that is compact, lightweight, and robust, that requires no high voltage (typically $20-70 \mathrm{~V}$ ). SiPMs have by now been shown by many groups to perform well as readout devices for scintillators ${ }^{10-13}$.

\footnotetext{
* Peter.Bloser@unh.edu; phone +1-603-862-0289; fax +1-603-862-3584

UV, X-Ray, and Gamma-Ray Space Instrumentation for Astronomy XVIII, edited by

Oswald H. Siegmund, Proc. of SPIE Vol. 8859, 88590A · @) 2013 SPIE

CCC code: $0277-786 X / 13 / \$ 18 \cdot$ doi: $10.1117 / 12.2024411$
} 
Commercial devices with sensitive areas of $1 \mathrm{~cm}^{2}$ or more are now available, as well as smaller devices designed to be easily tiled into larger arrays. Scintillator-based Compton cameras with SiPM readouts are already being developed by multiple groups for medical imaging applications ${ }^{14,15}$.

A few issues must be kept in mind, however. The radiation hardness of SiPMs is an area of active study ${ }^{16}$. In general, SiPMs show an order-of-magnitude increase in the single-cell dark count rate, but only small changes in pulse amplitude or PDE, after irradiation by fluences of $\sim 10^{10} 1-\mathrm{MeV}$-equivalent neutrons $\mathrm{cm}^{-2}$, corresponding roughly to several years in low earth orbit. Although more work is needed, this is likely acceptable for gamma-ray detectors using bright scintillators. For a given SiPM size, decreasing the number of cells decreases the relative fraction of dead area, and thus increases the geometric fraction and the PDE. There is thus a tradeoff between PDE and dynamic range, since a large dynamic range requires a large number of cells. The finite number of cells also leads to a saturation of the output signal for large energies, degrading energy resolution ${ }^{13}$. Cross talk between cells and after pulsing within cells can further worsen the resolution, though technical improvements are taking place ${ }^{17}$. Finally, the gain of the SiPM depends on the difference between the applied bias voltage and the breakdown voltage, which in turn depends on the temperature. Therefore, one must adjust the bias voltage to maintain stable performance.

The Space Science Center at the University of New Hampshire (UNH) is conducting a number of detector development programs based on the use of scintillators in high-energy astronomy and solar physics. For the past several years we have therefore been investigating the use of commercially available SiPMs in various scintillator readout configurations ${ }^{10,11}$. Most recently, we have flown a small detector consisting of a $6 \mathrm{~mm} \times 6 \mathrm{~mm} \times 10 \mathrm{~mm}$ $\mathrm{LaBr}_{3}$ crystal read out by a $6 \mathrm{~mm} \times 6 \mathrm{~mm}$ Hamamatsu S10985-050C SiPM as a piggyback payload on a scientific balloon flight ${ }^{12}$. The detector, located in a pressure vessel, performed well during 16 hours at float.

In this paper we report initial laboratory results obtained using larger scintillators and SiPM readouts, including gamma-ray spectroscopy with $\mathrm{LaBr}_{3}$ and PSD measurements with organic stilbene.

\section{GAMMA-RAY SPECTROSCOPY WITH LARGE-AREA SIPM ARRAY}

Because we are primarily interested in gamma-ray detectors based on $\mathrm{LaBr}_{3}$, we have limited our investigation to SiPMs with good sensitivity at blue wavelengths (for $\mathrm{LaBr}_{3}$ scintillation light, the peak wavelength $\lambda_{\text {peak }}=380 \mathrm{~nm}$ ). The largest commercially available blue-sensitive SiPM is the $13 \mathrm{~mm} \times 13 \mathrm{~mm}$ S11828-3344 MPPC from Hamamatsu Corp. (Fig. 1). This device is actually a $4 \times 4$ array of $3-\mathrm{mm}$ SiPMs on a single chip. The device features a total of 57600 cells, each $50 \mu \mathrm{m}$ in size, and an operating bias voltage of $\sim 72 \mathrm{~V}$.

The S11828-3344 SiPM was mounted on a custom carrier board using a solder reflow technique (Fig. 2). The board fabrication and SiPM mounting were performed by Intelligent Manufacturing Solutions Corp. (Manchester, $\mathrm{NH})$. A small thermistor was thermally coupled directly to the SiPM chip to permit the bias voltage to be adjusted based on the temperature to maintain a constant gain, as was successfully done in previous work ${ }^{12}$. For the work described here all 16 channels of the array were summed together within the connector on the carrier board.

A new, larger $\mathrm{LaBr}_{3}$ detector was purchased for this work from Saint-Gobain Crystals (Fig. 3). The scintillator crystal is $13 \mathrm{~mm} \times 13 \mathrm{~mm} \times 13 \mathrm{~mm}$, wrapped in white reflective material, and hermetically sealed in a thin

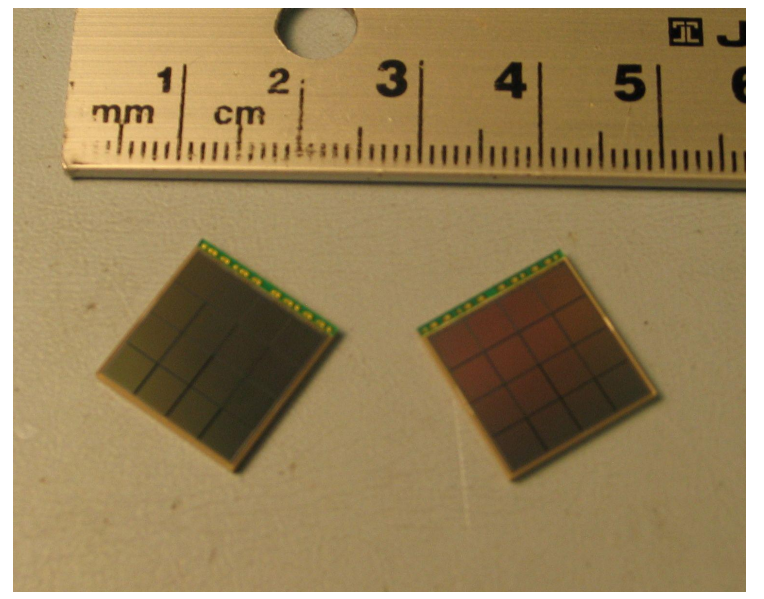

Figure 1: The S11828-3344 MPPC SiPM array from Hamamatsu Corp.

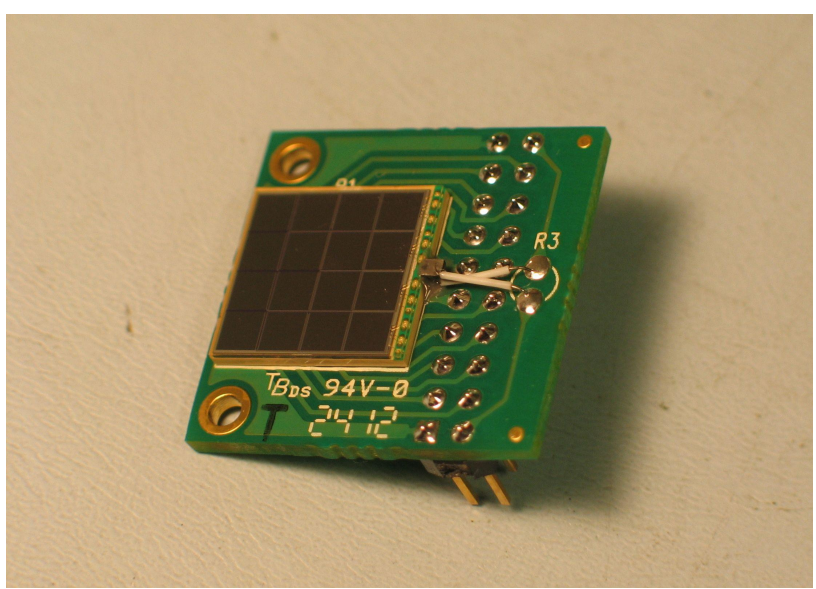

Figure 2: The S11828-3344 SiPM array mounted on a test readout board, with thermistor coupled directly to the SiPM chip. 


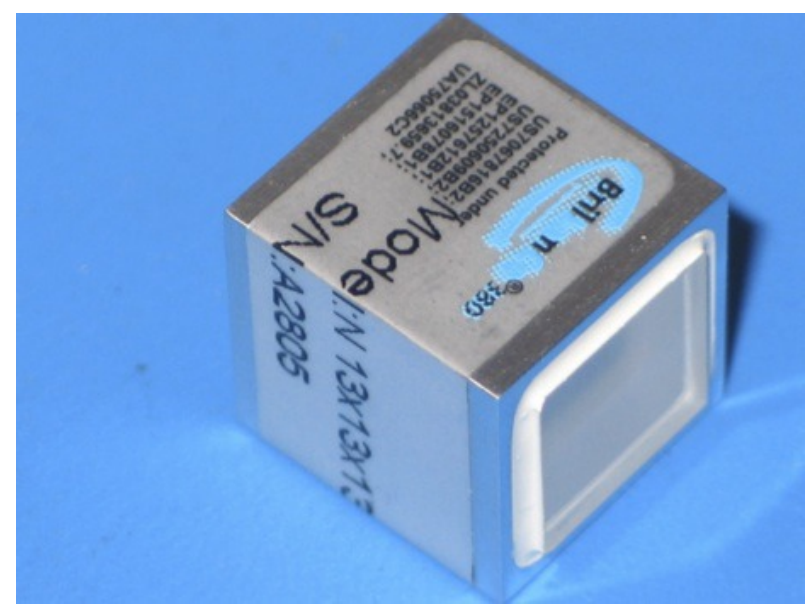

Figure 3: $13 \mathrm{~mm} \times 13 \mathrm{~mm} \times 13 \mathrm{~mm} \mathrm{LaBr} 3$ crystal with 3-mm light guide.

aluminum package with a 3-mm light guide on one face. The light guide was optically coupled to the S11828-3344 SiPM array using optical grease. Although the light guide will somewhat inhibit the collection of scintillation light, and thus slightly degrade the energy resolution, this configuration allows for much greater flexibility in laboratory testing.

It is critical for optimum performance with SiPMs that the input impedance of the preamplifier be as close to zero as possible, in order to avoid nonlinear behavior and maximize the speed of the output pulses ${ }^{18}$. This is due to the fact that the impedance of an individual cell changes dramatically when it is triggered, so that the output impedance of the SiPM depends on the total number of cells triggered. The rise time is particularly slowed for larger SiPMs due to their large capacitance. We have developed a novel front-end using a transformer to mimic a very low input impedance. Using this approach, the output pulses from the S11828-3344 have a rise time of $\sim 35 \mathrm{~ns}$, as opposed to $\sim 300 \mathrm{~ns}$ with the readout electronics used in the balloon flight $^{12}$. The output is standard $50 \mathrm{ohm}$, and was fed into a CAEN N568B spectroscopy amplifier in a VME rack. Data were acquired using a LabVIEW ${ }^{\mathrm{TM}}$ DAQ system.

Gamma-ray spectra were recorded at a variety of energies using sealed, uncollimated radioactive sources to study the linearity of the SiPM response and to measure the energy resolution as a function of energy. These data were recorded at a constant temperature of $22^{\circ} \mathrm{C}$. The photopeaks of the gamma-ray lines were fit in the recorded pulseheight histograms using a Gaussian function plus a continuous background. The photopeak centroid values are plotted vs. input line energy in Fig. 4. As expected, the pulse-height $(P H)$ vs. energy response is not linear due to the finite number of cells that contribute to the signal. The relationship is well described by a function of the form

$$
P H \approx N_{\text {fired }}=N_{\text {cells }}\left(1-\exp \left(-N_{\text {phot }} \times P D E / N_{\text {cells }}\right)\right),
$$

where $N_{\text {fired }}$ is the number of excited cells (proportional to the pulse height), $N_{\text {cells }}$ is the total number of cells in the $\mathrm{SiPM}$, and $N_{\text {phot }}$ is the number of incident scintillation photons (proportional to the absorbed energy).

Using this fit, the pulse height histograms were converted into energy spectra. The spectrum recorded for a ${ }^{137} \mathrm{Cs}$ source is shown in Fig. 5. The energy resolution measured at $662 \mathrm{keV}$ is $4.5 \pm 0.1 \%$ (FWHM). The low energy threshold is $\sim 20 \mathrm{keV}$. The energy resolution values derived from all source spectra are plotted as a function of energy in Fig. 6. Also shown for comparison are the energy resolution values obtained from the same $\mathrm{LaBr}_{3}$ detector using a 1-inch R1924A-100 PMT with a super bialkali (SBA) photocathode for the light readout. The

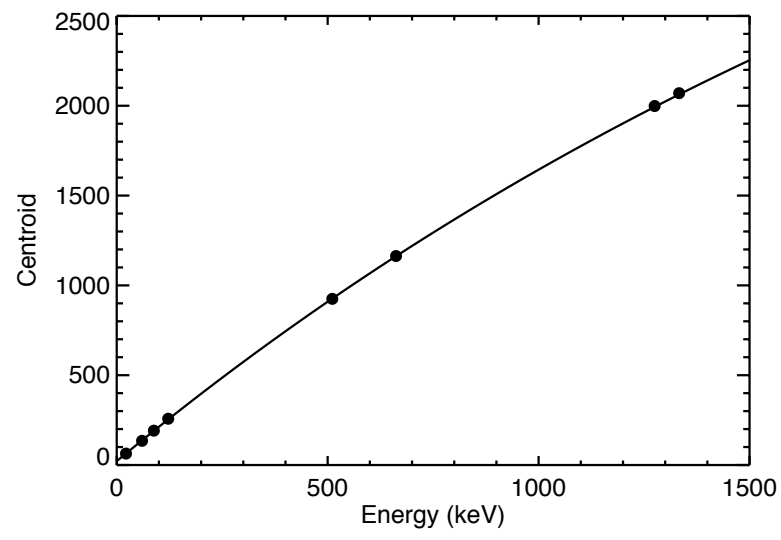

Figure 4: Pulse-height photopeak centroid vs. input energy for the $\mathrm{LaBr}_{3}$ crystal read out by the S11828-3344 SiPM. The expected non-linear relationship is observed and fit using Eq. 1.

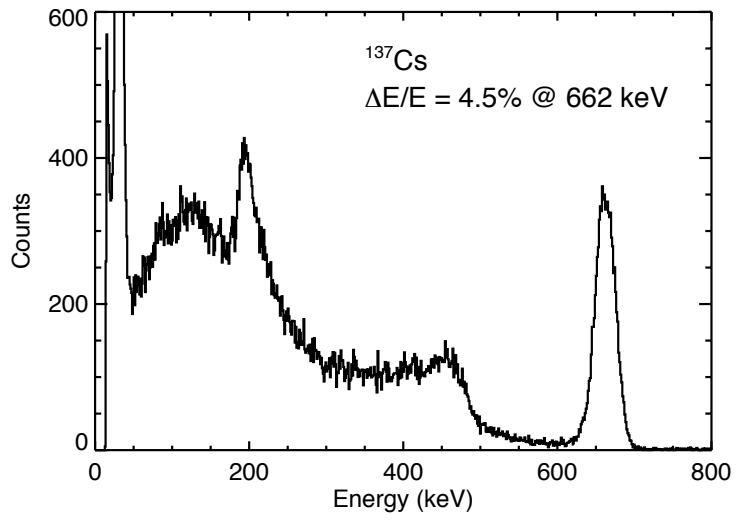

Figure 5: Energy spectrum of ${ }^{137} \mathrm{Cs}$ recorded with the new $\mathrm{LaBr}_{3}$ detector and S11828-3344 SiPM array. The energy resolution at $662 \mathrm{keV}$ is $4.5 \%$ (FWHM). 


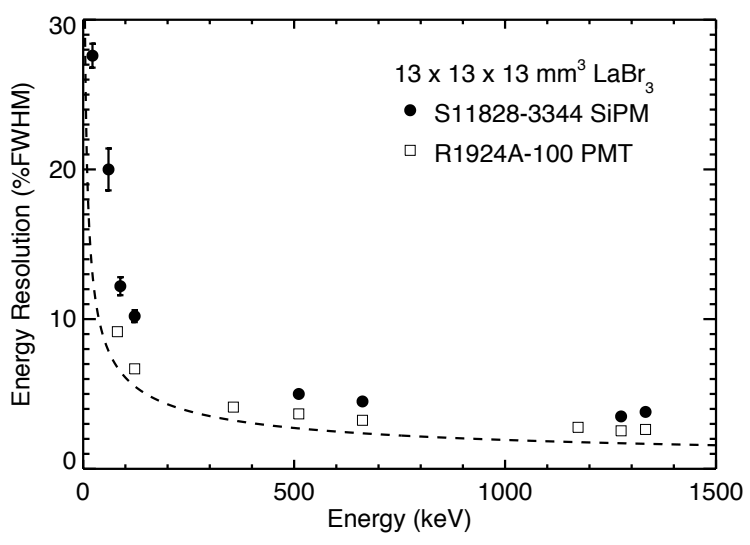

Figure 6: Energy resolution as a function of energy for a 13 $\mathrm{mm} \times 13 \mathrm{~mm} \times 13 \mathrm{~mm} \mathrm{LaBr} 3$ detector with 3 -mm light guide recorded by a the S11828-3344 SiPM array (circles) and a R1924A-100 PMT (squares). are of interest for space science applications, both as scattering detectors for Compton telescopes and as neutron detectors. Due to the different scintillation light decay times, signals from electrons (due to gamma-ray interactions) and protons (due to neutron scatters) may be differentiated via PSD ${ }^{6}$. As part of the Dose Spectra from Energetic particles and Neutrons (DoSEN) project at $\mathrm{UNH}^{20}$, we have investigated the use of SiPMs to identify neutron scatters in organic stilbene scintillator via PSD, in order to demonstrate the feasibility of compact dosimeters for fast $(\sim 1-100 \mathrm{MeV})$ neutrons in space.

Containing recoil protons with energies of $10 \mathrm{~s}$ of $\mathrm{MeV}$ requires a stilbene crystal at least 1 inch in size, and therefore necessitated the creation of a larger SiPM array. For the DoSEN prototype, we therefore formed a $26 \mathrm{~mm}$ $\times 26 \mathrm{~mm}$ light collecting area using a $2 \times 2$ array of S11828-3344 SiPMs. The four SiPMs were mounted on a custom array board (Fig. 7) that directly incorporates a transformer front-end for each chip. A single thermistor bead is included as well to monitor the temperature. Separate $50-\mathrm{ohm}$ outputs are provided for each quadrant of the array, which were summed for the measurement presented here. A 1-inch cylindrical stilbene crystal was purchased from Proteus, Inc., wrapped in white Teflon tape, and mounted on the SiPM array using optical grease (Fig. 8). A custom low-noise power supply provides a bias voltage of $\sim 72 \mathrm{~V}$ that automatically scales by $55 \mathrm{mV} /{ }^{\circ} \mathrm{C}$ to compensate for temperature-induced gain variations, using the thermistor on the carrier board as input.

To demonstrate experimentally that the SiPM readout could successfully distinguish between gamma-ray and neutron interactions via PSD, we exposed the stilbene to a ${ }^{252} \mathrm{Cf}$ fission source. The summed output signals from the SiPM array were fed into an Ortec 552 Pulse Shape Analyzer (PSA) module. The PSA outputs timing signals that

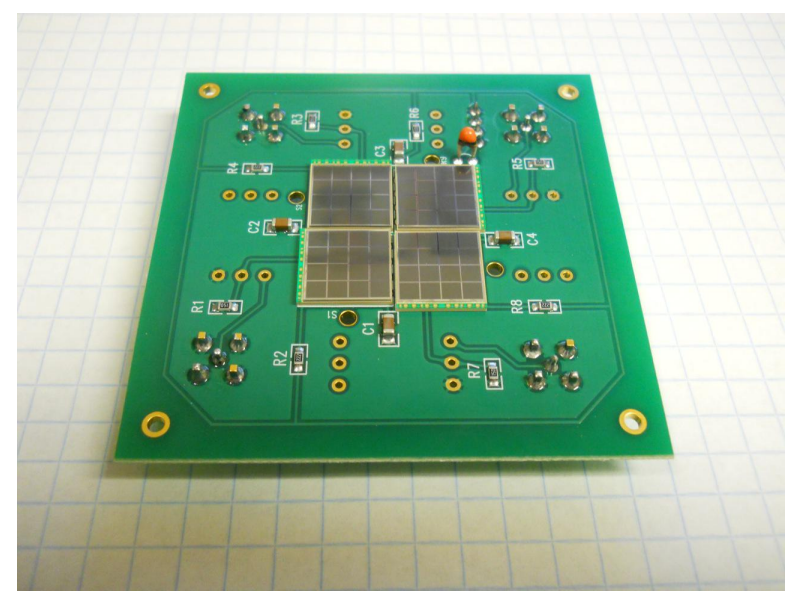

Figure 7: $2 \times 2$ array of Hamamatsu S11828-3344 SiPMs mounted on custom array board to form total $26 \mathrm{~mm} \times 26$ mm light collection area.

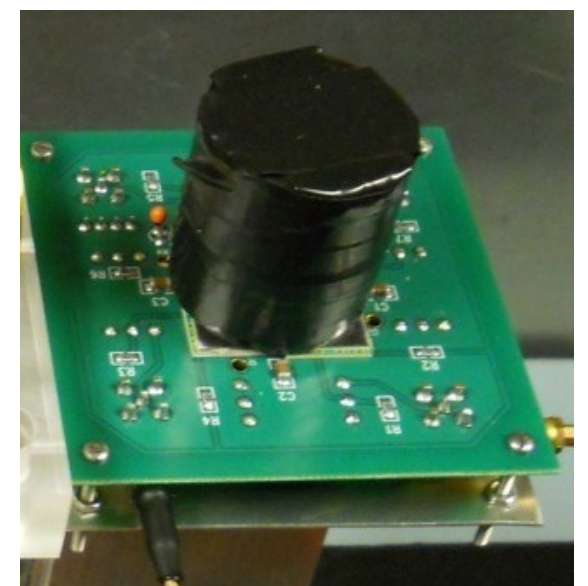

Figure 8: The 1-inch cylindrical stilbene crystal optically coupled to the $26 \mathrm{~mm} \times 26 \mathrm{~mm} \mathrm{SiPM}$ array as part of the DoSEN prototype. 
represent the difference between $20 \%$ and $80 \%$ decay fractions from the pulse peak. We refer to the ratio of these signals as the PSD parameter. The PSD vs. pulse height recorded for the ${ }^{252} \mathrm{Cf}$ source is shown in Fig. 9. The upper band of points is produced by recoil protons from fast neutron interactions, and the lower band by recoil electrons from gamma-ray Compton scatters. The bands are clearly separated, demonstrating that PSD can indeed be performed using organic scintillators with SiPM readouts.

An energy calibration was performed using Compton edges from laboratory gamma sources. The energy range in Fig. 9 is roughly $55 \mathrm{keV}$ to $3.3 \mathrm{MeV}$ for electrons. Using published light output calibrations for stilbene $^{21}$, this corresponds roughly to $0.5-8.2 \mathrm{MeV}$ for protons. This range was appropriate for maximizing the neutron signal from the ${ }^{252} \mathrm{Cf}$ source, and can be easily adjusted to higher values by reducing the gain of the detector system.

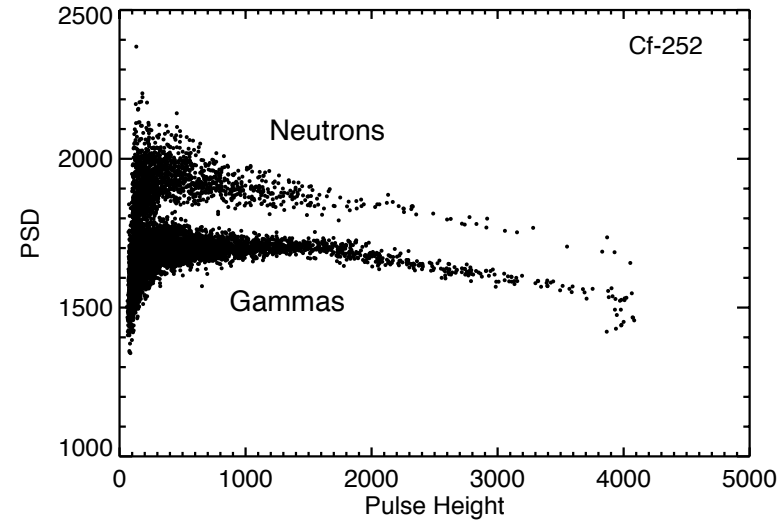

Figure 9: PSD vs. pulse height for the stilbene scintillator with $\mathrm{SiPM}$ readout. Fast neutrons and gamma rays from a ${ }^{252} \mathrm{Cf}$ source are clearly distinguished.

\section{GAMMA-RAY SPECTROSCOPY WITH ALTERNATE SIPMS}

Although the performance obtained using the Hamamatsu MPPCs is quite encouraging, we have begun investigating alternate SiPMs for use in gamma-ray spectroscopy with $\mathrm{LaBr}_{3}$. This is motivated primarily by the fast coincident timing requirements of a Compton telescope for gamma-ray astronomy ${ }^{7}$. Even using the transformer front end, the rise time obtained for the large-area S11828-3344 MPPC is 10s of ns, making it challenging to obtain sub-ns coincident timing. Recently, SensL Technologies Ltd. has introduced a new innovation that promises to greatly improve the timing response of SiPMs: the MicroFB-60035-SMT is a surface-mount, $6 \mathrm{~mm} \times 6 \mathrm{~mm}$, bluesensitive SiPM, designed for close packing, with a "fast output" capacitively coupled to each Geiger cell. This device promises $\sim$ ns rise times even for relatively large areas. In addition, the MicroFB-60035-SMT requires only $\sim 30 \mathrm{~V}$ bias voltage and is less sensitive to temperature variations $\left(\sim 20 \mathrm{mV} /{ }^{\circ} \mathrm{C}\right)$.

We have recently begun investigating the use of the MicroFB-60035-SMT SiPM for gamma-ray spectroscopy and timing. A $2 \times 2$ array was mounted on a custom carrier board (Fig. 10), creating a $\sim 13 \mathrm{~mm} \times 13 \mathrm{~mm}$ light collecting area equivalent to the Hamamatsu S11828-3344 MPPC and matched to our existing $\mathrm{LaBr}_{3}$ detector. The "fast" and "slow" outputs of the four devices were summed, and a transformer front-end was implemented for each. The rise time of the fast output was measured to be $\sim 2 \mathrm{~ns}$ using a blue LED, much faster than the $\sim 35 \mathrm{~ns}$ rise time of the MPPC of the same area.

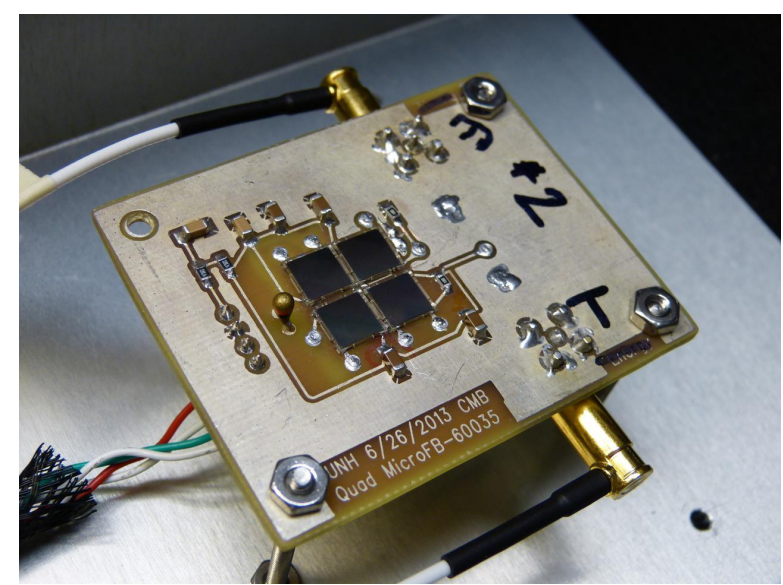

Figure 10: $2 \times 2$ array of SensL MicroFB-60035-SMT SiPMs on a custom carrier board. A transformer front end was implemented for both the "fast" and "slow" outputs.

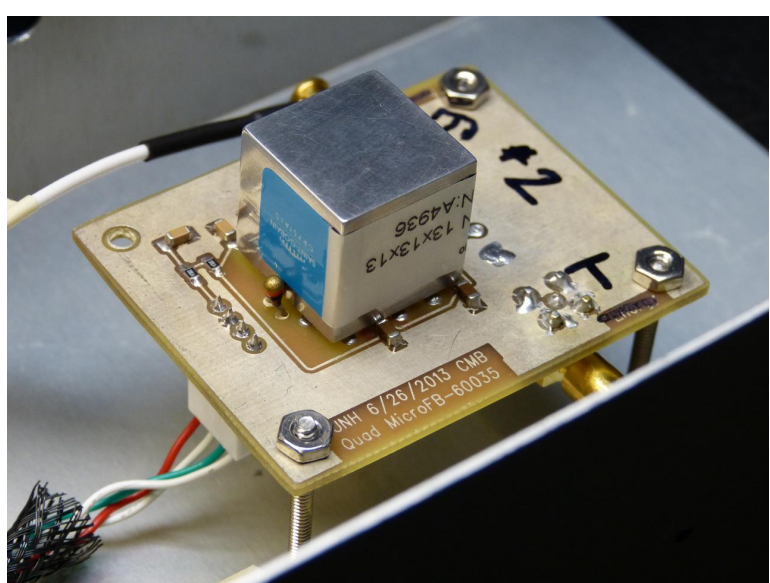

Figure 11: $13 \mathrm{~mm} \times 13 \mathrm{~mm} \times 13 \mathrm{~mm} \mathrm{LaBr} 3$ crystal mounted on the SensL SiPM array. 


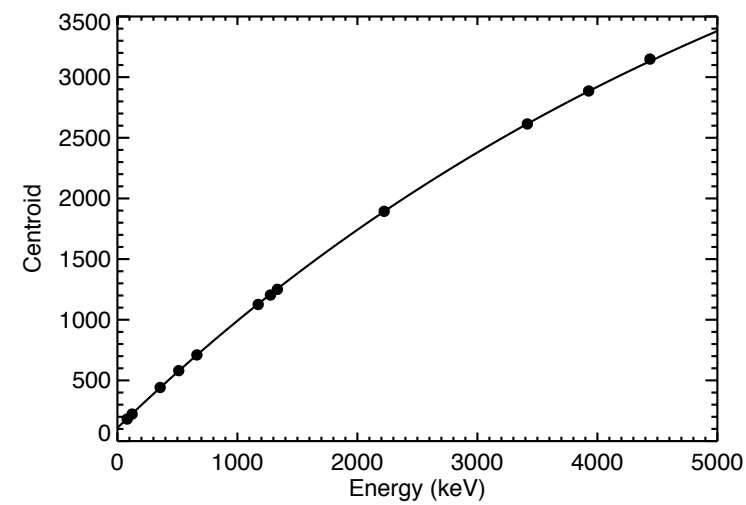

Figure 12: Pulse-height photopeak centroid vs. input energy for the $\mathrm{LaBr}_{3}$ crystal read out by the MicroFB-60035-SMT SiPM array, fit with Eq. 1 as in Fig. 4.

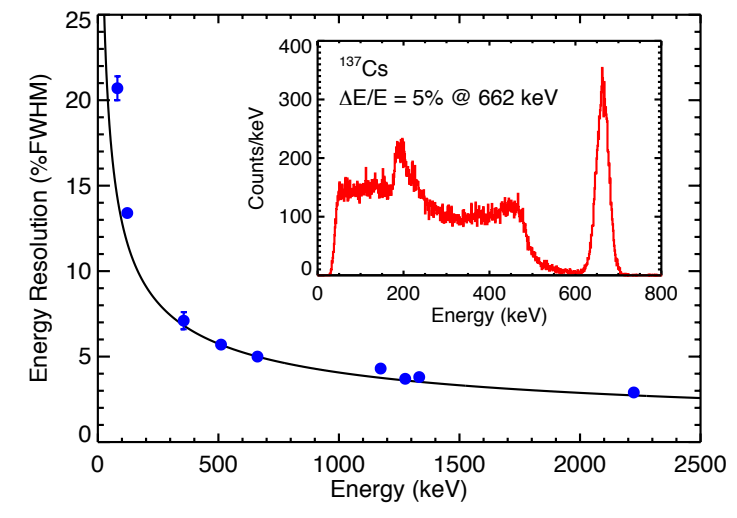

Figure 13: Energy resolution the $13 \mathrm{~mm} \times 13 \mathrm{~mm} \times 13 \mathrm{~mm}$ $\mathrm{LaBr}_{3}$ detector recorded by a the MicroFB-60035-SMT SiPM array, and spectrum of ${ }^{137} \mathrm{Cs}$ (inset).

The $13 \mathrm{~mm} \times 13 \mathrm{~mm} \times 13 \mathrm{~mm} \mathrm{LaBr} 3$ detector was coupled to the SensL SiPM array using optical grease (Fig. 11), and gamma-ray spectra were recorded as before (Sec. 2). We note that the scintillator could not be properly centered on the SiPM array due to the placement of a capacitor on the carrier board, so that the light collection was not ideal. Nevertheless, good spectra were obtained, and the centroids were fit as described in Sec. 2. In addition, higher-energy gamma-rays were produced by surrounding the detector with wax blocks and irradiating them with neutrons from a $50 \mathrm{mCi} \mathrm{Am} / \mathrm{Be}$ source. Neutron capture in the wax produced a $2223 \mathrm{keV}$ line, and the de-excitation of carbon produced in the $\mathrm{Am} / \mathrm{Be}$ source produced $4438 \mathrm{keV}$ gamma rays. A double escape peak was also observed at $3416 \mathrm{keV}$.

The pulse height centroid vs. energy is shown in Fig. 12. It shows the expected non-linearity, and is well-fit by Eq. 1. Using this calibration, the energy resolution as a function of energy was calculated using all lines up to 2223 $\mathrm{keV}$ (the $4438 \mathrm{keV}$ line is intrinsically broadened in the source). The result is shown in Fig. 13, along with the calibrated ${ }^{137} \mathrm{Cs}$ spectrum (inset). The energy resolution at $662 \mathrm{keV}$ is $5.0 \pm 0.1 \%$ (FWHM). This is slightly worse than the result obtained with the Hamamatsu MPPC, though we note again that the crystal could not be properly centered on the SensL array. The dynamic range is approximately $50 \mathrm{keV}$ to $6 \mathrm{MeV}$. The SensL MicroFB-60035SMT is thus another promising SiPM for gamma-ray astronomy applications.

\section{APPLICATION TO A SCINTILLATOR-BASED COMPTON TELESCOPE}

One potential application in gamma-ray astronomy where SiPMs could provide significant advantages is in a scintillatorbased Compton telescope. The only Compton telescope to perform sensitive astronomical observations in space was the COMPTEL instrument on $\mathrm{CGRO}^{22}$. COMPTEL employed liquid organic scintillator in a scattering detector layer (D1), and $\mathrm{NaI}$ inorganic scintillator in a calorimeter layer (D2). Neutron background was rejected via PSD in the scattering layer, and valid gamma-ray scatters were identified by measuring the time-of-flight (ToF) between the Compton scatter in D1 and the absorption in D2. Greatly improved response and background rejection would be possible in an updated instrument making use of $\mathrm{LaBr}_{3}$ in $\mathrm{D} 2$ to increase the energy and ToF resolution ${ }^{2,7}$. Replacing the liquid D1 with a brighter, solid crystal scintillator such as stilbene or pterphenyl would reduce complexity while further improving performance. However, the instrument would still be limited by the mass and volume of the PMT readouts.

We have begun investigating the use of SiPMs in a scintillatorbased Compton telescope to greatly reduce the mass and volume required for light readout. One possible configuration of a subsection of such an instrument is shown in Fig. 14. Each individual scintillator element would be read out by an array of
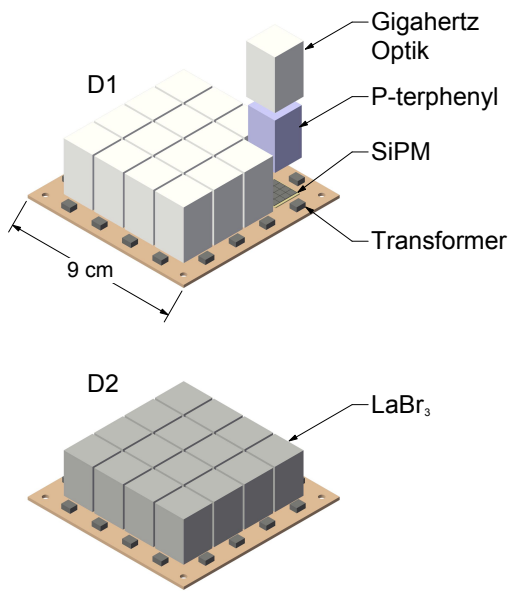

Figure 14: Concept for a subsection of a scintillator-based Compton telescope with SiPM readouts. 
SiPMs of the appropriate size. The SiPM arrays would be mounted on a carrier board with transformer front ends and temperature sensors incorporated. There would be very little passive mass near the detector units. Most importantly, very little mass would be placed between D1 and D2 detector layers. This would allow multiple layers of D1 and D2 detectors to be stacked in close proximity, increasing efficiency while maintaining small individual resolution elements (at the expense of increased channel count). If sub-ns ToF resolution can be realized, the separation between the D1 layers and D2 layers could be greatly reduced from the $\sim 1.5 \mathrm{~m}$ used in COMPTEL, increasing efficiency enormously while maintaining excellent background rejection. The energy resolution already obtained using $\mathrm{LaBr}_{3}$ with SiPM readouts is much better than that of COMPTEL $(\sim 10 \%$ at $1 \mathrm{MeV})$, and the PSD capability demonstrated using stilbene with SiPM readouts is equivalent to that of liquid scintillators with PMTs as used in COMPTEL. An advanced scintillator-based Compton telescope with SiPM readouts thus has the potential to significantly advance the field of $\mathrm{MeV}$ gamma-ray astronomy, which has languished since the demise of COMPTEL in 2000.

\section{SUMMARY}

SiPMs continue to show great potential as readout devices for advanced scintillators, permitting the design of innovative instruments for high-energy astronomy and space science. We will continue to advance the technical readiness of these devices in the hopes of soon flying new gamma-ray instrumentation on a balloon or satellite platform.

\section{ACKNOWLEDGMENTS}

This work was supported by NASA grants NNX10AG10G, NNX10AC14G, NNX12AB36G, and NNX13AC89G.

\section{REFERENCES}

1. van Loef, E. V. D., et al., "High Energy Resolution Scintillator: Ce ${ }^{3+}$ Activated LaBr3," Appl. Phys. Lett., 79, 1573 (2001).

2. Bloser, P. F., et al., "A fast scintillator Compton telescope for medium- energy gamma-ray astronomy," Proc. SPIE, 7732, $773222(2010)$.

3. Ertley, C., et al., "Simulations of a monolithic lanthanum bromide gamma-ray detector," Proc. SPIE, 7805, 78051X (2010).

4. Budden, B., Case, G. L., and Cherry, M. L., "Image reconstruction with a $\mathrm{LaBr}_{3}$-based rotational modulator," NIM-A, 652, 610 (2011).

5. Quarati, F. G. A., et al., "High energy gamma-ray spectroscopy with $\mathrm{LaBr}_{3}$ scintillation detectors," NIM-A, 629, 157 (2011).

6. Kim, H. D., et al., "Characteristics of a stilbene scintillation crystal in a neutron spectrometer," Rad. Meas., in press; doi:10.1016/j.radmeas.2013.01.004

7. Julien, M., et al., "Balloon flight results of a FAst Compton TELescope (FACTEL)," 2012 IEEE NSS/MIC Conference Record, 1893 (2012).

8. Buzhan, P., et al., "Silicon photomultiplier and its possible applications," Nucl. Inst. Meth. Phys. Res. A, 504, 48 (2003).

9. Schaart, D., et al., "LaBr3:Ce and SiPMs for time-of-flight PET: achieving 100 ps coincidence resolving time," Phys. Med. Biol., 55, N179 (2010).

10. Bloser, P. F., et al., "Silicon Photo-Multiplier Readouts for Scintillators in High-Energy Astronomy," 2008 IEEE NSS/MIC Conference Record, 727 (2008).

11. Bloser, P. F., et al., "Silicon Photo-Multiplier Readouts for Scintillator- Based Gamma-Ray Detectors in Space," 2010 IEEE NSS/MIC Conference Record, 357 (2010).

12. Bloser, P. F., et al., "Balloon-Flight Test of a Lanthanum Bromide Gamma-Ray Detector with Silicon Photomultiplier Readout," 2012 IEEE NSS/MIC Conference Record, 912 (2012).

13. Christian, J., et al., "Advances in CMOS solid-state photomultipliers for scintillation detector applications," NIM-A, 624, 449 (2010).

14. Saull, P. R. B., et al., "First demonstration of a Compton gamma imager based on silicon photomultipliers," NIM-A, 679, 89 (2012)

15. Llosá, G., et al., "Detector characterization and first coincidence tests of a Compton telescope based on $\mathrm{LaBr}_{3}$ and SiPMs," NIM-A, 695, 105 (2012).

16. Qiang, Y., et al., "Radiation hardness test of SiPMs for the JLab Hall D Barrel calorimeter," NIM-A, 698, 234 (2013). 
17. Dolgoshein, B., et al., "Large area UV SiPMs with extremely low cross-talk," NIM-A, 695,40 (2012).

18. Huizenga, J., et al., "A fast preamplifier concept for SiPM-based time-of-flight PET detectors," NIM-A, 695, 379 (2012).

19. Swiderski, L., et al., "Energy Resolution of Compton Electrons in $\mathrm{LaBr}_{3}$ :Ce Scintillator," IEEE Trans. Nucl. Sci., 57, 1697 (2010).

20. Schwadron, N., et al., "Dose Spectra from Energetic particles and Neutrons (DoSEN)," Space Weather, submitted.

21. Hansen, W., \& Richter, D., "Determination of light output function and angle dependent correction for a stilbene crystal scintillation neutron spectrometer," NIM-A, 476, 195 (2002).

22. Schönfelder, V., et al., "Instrument description and performance of the imaging gamma-ray telescope COMPTEL aboard the Compton Gamma-Ray Observatory,” Astrophys. Jour. Supp. Ser., 86, 657 (1993). 